\title{
Secure beamforming design for the UAV-enabled transmission over NOMA networks
}

\author{
Chuan Yin ${ }^{1}$ and Leibing Yan $^{2 *}$
}

\begin{abstract}
This paper considers an unmanned aerial vehicle -enabled multicast-unicast transmit system, in which downlink non-orthogonal multiple access (NOMA) is applied. Since two types of information are broadcast simultaneously, the security of information transmission is a matter of concern. Based on this, we investigate the problem of maximizing the secrecy rate of the particular unicasting user subject to the quality-of-service (QoS) requirements of the multicasting users and the power budget constraint of the UAV base station (BS). Such a problem is non-convex and hard to tackle, and a two-stage procedure is proposed to solve it optimally by dealing with a sequence of semidefinite relaxation (SDR) counterparts. First, fixing the maximal signal to interference plus noise ratio (SINR) of unicasting information in other users, we obtain the corresponding SINR of unicasting information at the particular user. Then, the maximal secrecy unicasting rate is derived by a one-dimension search over the intercepted SINR of unicasting information in other users. Furthermore, two suboptimal solutions with lower complexity are also proposed. Numerical simulation shows that the proposed NOMA assisted schemes always have a better security performance for UAV-enabled transmission.
\end{abstract}

Keywords: Unmanned aerial vehicles, Mobile base station, Non-orthogonal multiple access, Quality-of-service

\section{Introduction}

More and more techniques are proposed to improve network capacity and spectral efficiency [1-3]. As a promising technology, non-orthogonal multiple access (NOMA) has attracted a lot of research interests due to its superior spectral efficiency [4-7]. Unlike conventional orthogonal multiple access (OMA), NOMA serves multiple users with different power levels at the same time, code and frequency $[8,9]$, where successive interference cancellation (SIC) is employed at users. Many different kinds of NOMA designs, combined with wireless power supply [10-12], millimeter-wave communication [13], and mobile edge computing [14] have appeared in recent researches. Cognitive radio and NOMA are also integrated to improve spectral efficiency and increase system capacity [15].

*Correspondence: hngxyylb@126.com

${ }^{2} S c h o o l$ of Electronic Information Engineering, Henan Institute of Technology, 453003, Xinxiang, China

Full list of author information is available at the end of the article
Although NOMA can achieve promising performance, there still exist challenges, especially when it is used to provide massive connections. Firstly, the quality of service (QoS) of edge users needs to be further improved [16]. In addition, more users should be served in a single subcarrier. This may increase the load and delay of SIC [17]. For these reasons, unmanned aerial vehicles (UAVs) can be exploited as a potential method to assist NOMA to achieve better performance. Due to the flexibility and mobility, UAVs have drawn increasing attention, which can be applied to many wireless scenarios including ubiquitous coverage, relaying, as well as information dissemination and data collection [18].

Recently, plenty of excellent research on UAV communications has been conducted. The authors of [19] considered a UAV-BS to communicate with two ground users using NOMA and investigated their outage probability. In [20], the authors characterized the capacity region of a UAV-enabled broadcast channel with two 
ground users and jointly optimized the UAV's trajectory and transmit power/rate allocations over time. In [21], the authors employed a UAV system and NOMA to optimize the power allocation and the UAV altitude to maximize sum-rate for two users [22, 23]. However, in order to achieve the maximum rate gains from UAV-enabled communication, it is important to jointly optimize multiple relevant parameters, e.g., UAV altitude, antenna beamwidth, power allocation, and bandwidth allocation. To the authors' best knowledge, this important problem, within a NOMA setting, has not been treated previously.

It is worth noting that a signal bearing different information messages sent from the UAV-BS is transmitted to corresponding receivers, which is equivalent to unicasting scenarios. So the aforementioned research efforts focus on wireless unicast strategies. However, if some users have the common interest, the same data hence has to be sent separately, which would lead to low spectral efficiency and inefficient utilization. Considering the broadcasting feature of wireless channel, multicast can deliver the same information to all users, while unicasting stream can be transmitted to one user within the same resource block [24], which yields bandwidth saving and efficient utilization.

Meanwhile, beamforming is a signal processing technique used in various wireless systems for directional communications and security communication [25], rather than information theoretic aspect [26, 27]. The integration of NOMA and multi-user beamforming thus has the potential to capture the benefits of both NOMA and beamforming $[28,29]$. Therefore, in NOMA systems, when some users have the same interest, integrating multicast-unicast into NOMA can combine their advantages, and thus increase system capacity and reliability, boost the spectral efficiency and ameliorate link utilization simultaneously. The application of NOMA to multicasting transmission has also drawn some attention [24, 30]. The work of [30] has designed a beamforming-based NOMA unicast-multicast strategy, which has employed beamforming and power allocation to improve the reliability of a unicasting user while guaranteeing the quality of a group of multicasting users. However, the maximal ratio transmission (MRT) principle is applied in [30], which does not take full advantage of beamforming. Similar to NOMA protocol, [24] considers a multiple-antenna communication system with a mixed unicast and multicast traffic, where the successive decoding is applied at the unicast user to decode both common and private messages, while only the common message will be decoded at the other user. But this work focuses on two-user case of multicast, and that case is also considered in [31], whereas, in practical networks, a large number of users may intend the same data due to common interest. The problem of transmitting beamforming to multiple co-channel multicast groups based on semidefinite relaxation (SDR) has been proposed in [32] without the consideration of unicast.

The remainder of this paper is organized as follows. Section 2 proposes the main method for this paper. Section 3 introduces the system model of the NOMAenabled multicast-unicast system and then presents the formulated optimization problem. In Section 4, the optimal solution to the problem is proposed. Section 5 presents two suboptimal solutions for the problem. Numerical results are provided to compare the performances of various proposed schemes in Section 6. The conclusions are given in Section 7.

\subsection{Notations}

Scalar is denoted by lower-cases letters, vectors are denoted by boldface lower-case letters, and matrices are denoted by boldface upper-case letters. For a square matrix $\mathbf{A}, \operatorname{tr}(\mathbf{A}), \operatorname{rank}(\mathbf{A})$ and $\mathbf{A}^{H}$ denote its trace, rank, and conjugate, respectively. $\mathbf{A} \geq 0$ and $\mathbf{A} \leq 0$ represent that $\mathbf{A}$ is a positive semidefinite matrix and a negative semidefinite matrix, respectively. $\|\mathbf{x}\|$ denotes the Euclidean norm of a complex vector $\mathbf{x} . E[\cdot]$ denotes the statistical expectation. The distribution of a circularly symmetric complex Gaussian (CSCG) random vector with mean vector $\mathbf{x}$ and covariance matrix $\Sigma$ is denoted by $C N(0, \Sigma)$, and $\sim$ stands for "distributed as". $\mathbb{C}^{x \times y}$ denotes the space of $x \times y$ complex matrices.

\section{Method}

In this paper, we consider a physical layer security problem in a multiuser network with mixed multicast and unicast information streams, which are superposed and simultaneously sent from the UAV-BS. The multicasting information is for every user while the unicasting information is for the special one of the users. Actually, the cognitive radio concept is used by assigning multicasting message with a higher priority compared to unicasting message. Thus, all users can first apply SIC to decode the multicast and then subtract it from their observations before the unicast is decoded. So the unicasting information may be intercepted by the other users, who are potential eavesdroppers. Different from [30], we aim to maximize the secrecy rate of the unicasting signal via the design of proper beamforming vectors for different users, subject to the users' quality of service (QoS) about multicasting information and the power constraint of the BS, which is solved by a two-stage procedure. Since all the users' channel state information (CSI) are perfectly available at the BS, the artificial noise (AN) precoding technologies are not required, and AN-aided beamforming also achieves an inherent trade-off between the transmission rate and the ability to impair eavesdroppers [33]. Although [34] considered the AN-aided transmit beamforming, where the channels of two eavesdroppers could 
be considered as multicast transmission in some cases, the maximum allowable signal to interference plus noise ratio (SINR) threshold on eavesdroppers is given. As a matter of fact, a more practical model is considered in this paper , in which neither the number of other users nor the maximal intercepted SINR of unicasting information is known. Furthermore, two suboptimal solutions with lower complexity are proposed. Finally, we compare the performance of our proposed solutions by numerical simulations.

\section{System model and problem formulation}

Consider the application of NOMA to a multiuser downlink communication system, in which one BS has two combined messages to send. We denote the multicasting message to all users by $s_{M}$ and denote the unicasting message to the certain user by $s_{U}$. The multicasting information $s_{M}$ can be received by all the users and the unicasting message $s_{U}$ is intended for the particular user, as shown in Fig. 1. The BS is equipped $N$ antennas and each user has a single antenna for the facility cost. Meanwhile, the user of the next generation wireless mobile communication systems also needs to satisfy the demand of low power consumption and portability, which results that using single receive antenna for each user would be more appropriate. It is assumed that the users' channels are quasi-static fading channels, and the CSI is perfectly available at the $\mathrm{BS}$.

It is assumed that $s_{M}$ and $s_{U}$ are independent and identically distributed (i.i.d.) CSCG random variables with unit average power, denoted by $s_{M}, s_{U} \sim C N(0,1)$. Therefore, the complex baseband transmitted signal of the BS can be expressed as:

$$
\mathbf{x}=\mathbf{w}_{0} s_{M}+\mathbf{w}_{1} s_{U}
$$

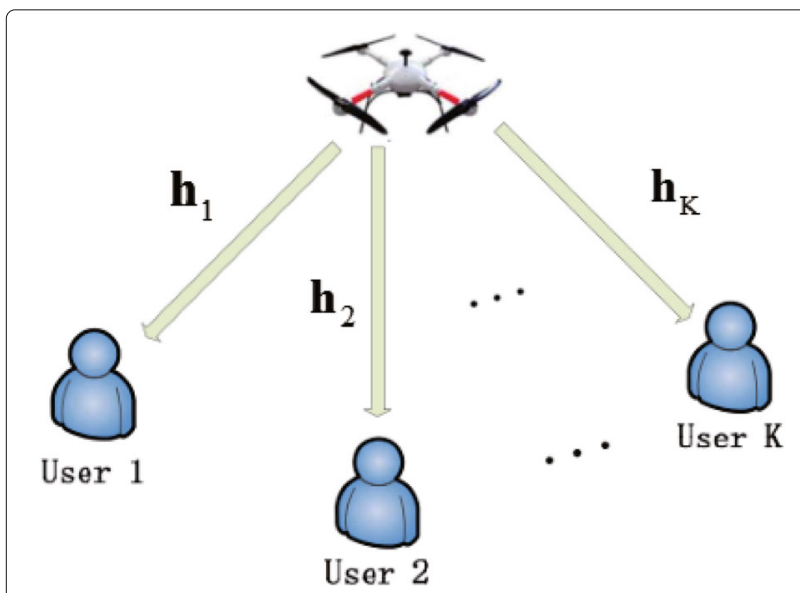

Fig. 1 A NOMA-enabled multicast-unicast transmission system where $\mathbf{w}_{0}, \mathbf{w}_{1} \in \mathbb{C}^{N \times 1}$ are multicasting and unicasting beamforming vectors, respectively. Suppose that the maximal transmission power budget of the BS is $P$. From [35], we thus have

$$
E\left[\mathbf{x}^{H} \mathbf{x}\right]=\left\|\mathbf{w}_{0}\right\|^{2}+\left\|\mathbf{w}_{1}\right\|^{2} \leq P
$$

The small scale-fading channel between the UAV and the ground users can be represented by a complex vector. In this paper, the matrix element $h_{p}(\tau)$ denotes the channel impulse response (CIR) between the $p$ th UAV antenna and the given ground user, and it can be expressed by the summation of the LoS component and several NLoS components as

$$
\begin{aligned}
h_{p}(\tau)= & \sqrt{\frac{K}{1+K}} h_{p}^{\mathrm{LoS}} \delta\left(\tau-\tau_{p}^{\mathrm{LoS}}\right)+\sqrt{\frac{1}{1+K}} \sum_{n=1}^{N} \\
& \sqrt{P_{p, n}} h_{p, n}^{\mathrm{NLoS}} \delta\left(\tau-\tau_{p, n}^{\mathrm{NLoS}}\right),
\end{aligned}
$$

where $N$ is the number of NLoS components, $K$ denotes the Ricean factor, $h_{p, n}^{\mathrm{NLoS}}, P_{p, n}$ and $\tau_{p}^{\mathrm{LoS}}$ mean the complex channel coefficient, the power and delay of the $n$th NLoS component, respectively, $h_{p, n}^{\mathrm{NLoS}}$ and $\tau_{p, n}^{\mathrm{NLOS}}$ represent the complex channel coefficient and the delay of the LoS component. It should be mentioned that this paper only considers the power-normalized small scale-fading channel model. The total power equals to one, while the power of the LoS component and all NLoS components are $\frac{K}{1+K}$ and $\frac{1}{1+K}$, respectively.

The observation at the $k$ th user is given by:

$$
y_{k}=\mathbf{h}_{k}^{H} \mathbf{x}+n_{i}=\mathbf{h}_{k}^{H}\left(\mathbf{w}_{0} s_{M}+\mathbf{w}_{1} s_{U}\right)+n_{k},
$$

where $\mathbf{h}_{k} \in \mathbb{C}^{N \times 1}, k=1, \cdots, K$, denotes the conjugated complex channel vector of user $k$. which is perfectly known at the BS. $n_{k}$ is additive Gaussian noise at each user, satisfying $n_{k} \sim C N\left(0, \sigma^{2}\right), k=1, \cdots, K$. Similar to conventional NOMA protocol, SIC can be carried out at all users, so all users can detect $s_{M}$ first and then subtract $s_{M}$ from the observations before $s_{U}$ is decoded. In fact, the cognitive radio concept is used here, which means that $s_{M}$ is assigned with higher priority compared to $s_{U}$. So the multicasting rate at each user can be written as $R_{k, M}=$ $\log _{2}\left(1+\operatorname{SINR}_{k, M}\right), k=1, \cdots, K$ and the other users are potential eavesdroppers to intercept the unicasting information. So the secrecy rate of unicasting information is written as:

$$
R=\log _{2}\left(1+\operatorname{SINR}_{1, U}\right)-\max _{k=2}^{K} \log _{2}\left(1+\operatorname{SINR}_{k, U}\right)
$$

where

$$
\begin{aligned}
& \operatorname{SINR}_{k, M}=\frac{\left|\mathbf{h}_{k}^{H} \mathbf{w}_{0}\right|^{2}}{\left|\mathbf{h}_{k}^{H} \mathbf{w}_{1}\right|^{2}+\sigma^{2}}, k=1, \cdots, K . \\
& \operatorname{SINR}_{k, U}=\frac{\left|\mathbf{h}_{k}^{H} \mathbf{w}_{1}\right|^{2}}{\sigma^{2}}, k=1, \cdots, K .
\end{aligned}
$$


On the right side of (5), the first part represents the unicasting SINR at particular user, and the last part is the intercepted unicasting SINR, which is inevitable and unexpected. The secrecy rate of unicasting information is effective, when the particular user has a maximal unicasting SINR, including that user 1 has a better channel condition than the other users. Thus, prior to the NOMA transmission, the BS selects the user who has the maximal unicasting SINR in order to guarantee an effective secrecy unicasting rate. Here, we assume that the BS chooses the proper user as the particular user before transmission.

In this paper, we aim to maximize the secrecy rate of $s_{U}$ subject to the predefined QoS of $s_{M}$ at each user and the given transmission sum-power $P$ of the BS. The optimization problem can be formulated as:

$$
\begin{array}{ll}
\max _{\mathbf{w}_{0}, \mathbf{w}_{1}} & R \\
\text { s.t. } & \operatorname{SINR}_{k, M} \geq \gamma_{M}, k=1, \cdots, K, \\
& \left\|\mathbf{w}_{0}\right\|^{2}+\left\|\mathbf{w}_{1}\right\|^{2} \leq P,
\end{array}
$$

where $\gamma_{M}=2^{2 R_{M}-1}$ is the minimum SINR threshold for multicasting to satisfy the corresponding target rate requirement $R_{M}$.

\section{The optimal solution}

In this section, we propose an algorithm based on SDR [36] to solve the problem (8). As we can see, there always exists a maximal intercepted unicasting SINR $\gamma_{e}>0$ at other users except user 1 in (8a), i.e., $\max _{k=2}^{K} \operatorname{SINR}_{k, U} \leq$ $\gamma_{e}$. For given $\gamma_{e}>0$, the following problem (9) has the same optimal solution to problem (8) [37]:

$$
\begin{array}{ll}
\max _{\mathbf{w}_{0}, \mathbf{w}_{1}} & \left|\mathbf{h}_{1}^{H} \mathbf{w}_{1}\right|^{2} \\
\text { s.t. } & \operatorname{SINR}_{k, U} \leq \gamma_{e}, k=2, \cdots, K, \\
& \operatorname{SINR}_{k, M} \geq \gamma_{M}, k=1, \cdots, K, \\
& \left\|\mathbf{w}_{0}\right\|^{2}+\left\|\mathbf{w}_{1}\right\|^{2} \leq P,
\end{array}
$$

Let $f\left(\gamma_{e}\right)$ denote the optimal value of problem (9) with given $\gamma_{e}>0$, and then (8a) can be written as following:

$$
\begin{aligned}
R & =\log _{2}\left(1+\frac{f\left(\gamma_{e}\right)}{\sigma^{2}}\right)-\log _{2}\left(1+\gamma_{e}\right) \\
& =\log _{2}\left[\frac{\sigma^{2}+f\left(\gamma_{e}\right)}{\sigma^{2}\left(1+\gamma_{e}\right)}\right]=R\left(\gamma_{e}\right)
\end{aligned}
$$

It can be shown that the optimal value of problem (8) is the same as that of the following problem:

$$
\max _{\gamma_{e}>0} R\left(\gamma_{e}\right)
$$

Assume that $\gamma_{e}^{*}$ is the optimal solution to problem (11). From the above results, when $\gamma_{e}=\gamma_{e}^{*}$, problem (9) and
(8) have the same optimal solution. Hence problem (8) can be solved by two steps: First, given any $\gamma_{e}, f\left(\gamma_{e}\right)$ can be obtained by solving (9). Then, the optimal $\gamma_{e}^{*}$ for (8) can be obtained by one dimension search over $\gamma_{e}>0$. Therefore, in the rest of this section, we focus on solving (9).

Define $\mathbf{W}_{1}=\mathbf{w}_{1} \mathbf{w}_{1}^{H}, \mathbf{W}_{0}=\mathbf{w}_{0} \mathbf{w}_{0}^{H}$ and $\mathbf{H}_{k}=\mathbf{h}_{k} \mathbf{h}_{k}^{H}, k=$ $1, \cdots, K$. By ignoring the rank-one constraint on $\mathbf{W}_{1}$ and $\mathbf{W}_{0}$, the SDR of (9) can be expressed as:

$$
\begin{aligned}
& \max _{\mathbf{W}_{0} \geq 0, \mathbf{W}_{1} \geq 0} \operatorname{tr}\left(\mathbf{H}_{1} \mathbf{W}_{1}\right) \\
& \text { s.t. } \operatorname{tr}\left(\mathbf{H}_{k} \mathbf{W}_{1}\right) \leq \gamma_{\mathrm{e}} \sigma^{2}, k=2, \cdots, K
\end{aligned}
$$

$$
\operatorname{tr}\left(\mathbf{H}_{k} \mathbf{W}_{0}\right) \geq \gamma_{\mathrm{M}}\left[\operatorname{tr}\left(\mathbf{H}_{k} \mathbf{W}_{1}\right)+\sigma^{2}\right], k=1, \cdots, K
$$

$$
\operatorname{tr}\left(\mathbf{W}_{0}\right)+\operatorname{tr}\left(\mathbf{W}_{1}\right) \leq \mathrm{P},
$$

Obviously, problem (12) is a separable semidefinite program (SDP). According to Theorem 3.2 in [38], the optimal solution $\left(\mathbf{W}_{1}^{*}, \mathbf{W}_{0}^{*}\right)$ to problem (12) must satisfy $\operatorname{rank}\left(\mathbf{W}_{1}^{*}\right)+\operatorname{rank}\left(\mathbf{W}_{0}^{*}\right) \leq 2 K$, where $2 K$ denotes the number of linear constraints given in (12b)-(12d). But it is not sufficient to verify the rank-one of the variables because $K$ in the problem (12) may be very large. As a result, the result of [5] for separable SDPs cannot be applied in our problem (9) to show the tightness of SDP in (12).

Then, we will discuss the rank of the optimal solution $\mathbf{W}_{1}^{*}$ by the Langrangian dual method. Since problem (12) is convex and satisfies the Slater's condition, its duality gap is zero [39]. Let $\left\{\alpha_{k}\right\},\left\{\beta_{k}\right\}$ and $\lambda$ denote the dual variables of (12a), (12b), and (12c), respectively. Then, the Lagrangian function of problem (12) is expressed as:

$$
L\left(\mathbf{W}_{1}, \mathbf{W}_{0},\left\{\alpha_{k}\right\},\left\{\beta_{k}\right\}, \lambda\right)=\operatorname{tr}\left(\mathbf{A} \mathbf{W}_{1}\right)+\operatorname{tr}\left(\mathbf{B} \mathbf{W}_{0}\right)+\mathbf{C}
$$

where

$$
\begin{aligned}
& \mathbf{A}=\mathbf{H}_{1}-\sum_{k=2}^{K} \alpha_{k} \mathbf{H}_{k}-\gamma_{M} \sum_{k=1}^{K} \beta_{k} \mathbf{H}_{k}-\lambda \mathbf{I}, \\
& \mathbf{B}=\sum_{k=1}^{K} \beta_{k} \mathbf{H}_{k}-\lambda \mathbf{I}, \\
& \mathbf{C}=\gamma_{e} \sigma^{2} \sum_{k=2}^{K} \alpha_{k}-\gamma_{M} \sigma^{2} \sum_{k=1}^{K} \beta_{k}+\lambda P
\end{aligned}
$$

And the dual problem of (12) can be expressed as:

$$
\begin{aligned}
& \min _{\left\{\alpha_{k}\right\},\left\{\beta_{k}\right\}, \lambda} \gamma_{e} \sigma^{2} \sum_{k=2}^{K} \alpha_{k}-\gamma_{M} \sigma^{2} \sum_{k=1}^{K} \beta_{k}+\lambda P \\
& \text { s.t. } \boldsymbol{A} \leq 0, \boldsymbol{B} \leq 0,
\end{aligned}
$$




$$
\begin{aligned}
& \alpha_{k} \geq 0, \beta_{k} \geq 0, \\
& \lambda \geq 0,
\end{aligned}
$$

Denote the optimal dual variables of problem (17) as $\left(\left\{\alpha_{k}^{*}\right\},\left\{\beta_{k}^{*}\right\}, \lambda^{*}\right)$, and let $\mathbf{A}^{*}$ and $\mathbf{B}^{*}$ be given in (14) and (15), respectively. By substituting the optimal dual variables, we have the following proposition:

Proposition 1 The optimal dual solution to problem (12) satisfies that $\lambda^{*}>0$ when $\gamma_{e}>0$.

Proof We show that $\lambda^{*}>0$ by contradiction. Define $\Phi=\left\{k \mid\left(\alpha_{k}^{*}\right)^{2}+\left(\beta_{k}^{*}\right)^{2}>0, k=1, \cdots, K\right\}$; here define $\alpha_{1}^{*}=0$. Next, we will discuss that in the following two cases, the constraints of (17) cannot be satisfied when $\lambda^{*}=0$.

1. If $\Phi=\phi$ : In this case, we can derive that $\alpha_{k}^{*}=0, \beta_{k}^{*}=$ 0 . So the objective function of (9) is zero and the secrecy rate is zero. That is not true.

2. If $\Phi \neq \phi$ : According to the contraint of (17b), $\mathbf{B}^{*}=$ $\sum_{k \in \Phi} \beta_{k}^{*} \mathbf{H}_{k} \leq 0$, so $\beta_{k}^{*}=0$. Then, there must exists $k$ and $\alpha_{k}^{*} \neq 0$. It is obvious that $\sum_{k \in \Phi} \alpha_{k}^{*} \mathbf{H}_{k} \geq 0$. To guarantee that $\mathbf{A}^{*}=\mathbf{H}_{1}-\sum_{k \in \Phi} \alpha_{k}^{*} \mathbf{H}_{k} \leq 0$ and $\mathbf{A}^{*} \mathbf{W}_{1}^{*}=0$, it requires that the optimal solution $\mathbf{W}_{1}^{*}$, which lies in the null space of $\mathbf{H}_{k}$, must also lie in the null space of $\mathbf{H}_{1}$. However, in such case, user 1 cannot receive the unicasting information either. So in this case, we can also conclude that $\lambda^{*}>0$.

By combining the above two cases, we can have $\lambda^{*}>0$. The Proposition 1 is proved.

Due to the complementary slackness, in the optimal solution of (12), the sum power constraint must be satisfied with equality. Define

$$
\mathbf{D}^{*}=-\sum_{k=2}^{K} \alpha_{k}^{*} \mathbf{H}_{k}-\gamma_{M} \sum_{k=1}^{K} \beta_{k}^{*} \mathbf{H}_{k}-\lambda^{*} \mathbf{I}=\mathbf{A}^{*}-\mathbf{H}_{1}
$$

and $l=\operatorname{rank}\left(\mathbf{D}^{*}\right)$. Then, let $\Pi \in \mathbb{C}^{N \times(N-l)}$ denote the orthogonal basis of the null space of $\mathbf{D}^{*}$, where $\Pi=0$ if $l=N$, and $\pi_{n}$ denote the $n$th column of $\Pi, 1 \leq n \leq N-l$. Hence we have the following proposition.

Proposition 2 The optimal solution $\left(\mathbf{W}_{1}^{*}, \mathbf{W}_{0}^{*}\right)$ to (12) satisfies the following conditions:

1. $\mathbf{W}_{1}^{*}$ can be expressed as

$$
\mathbf{W}_{1}^{*}=\sum_{k=1}^{N-l} a_{n} \boldsymbol{\pi}_{n} \boldsymbol{\pi}_{n}^{H}+b \boldsymbol{\tau} \boldsymbol{\tau}^{H}
$$

where $a_{n} \geq 0, b>0$ and $\boldsymbol{\tau} \in \mathbb{C}^{N \times 1}$ has unit-norm and satisfies $\boldsymbol{\tau}^{\bar{H}} \Pi=0$.
2. If $\mathbf{W}_{1}^{*}$ given in (19) has the rank larger than one, there exists at least an $n$ such that $a_{n} \geq 0$, and the following solution with $\operatorname{rank}\left(\hat{\mathbf{W}}_{1}^{*}\right)=1$, is also an optimal solution to (12).

$$
\begin{aligned}
& \hat{\mathbf{W}}_{1}^{*}=b \boldsymbol{\tau} \boldsymbol{\tau}^{H} \\
& \hat{\mathbf{W}}_{0}^{*}=\mathbf{W}_{0}^{*}+\sum_{k=1}^{N-l} a_{n} \boldsymbol{\pi}_{n} \boldsymbol{\pi}_{n}^{H}
\end{aligned}
$$

Proof If $l=N, \operatorname{rank}\left(\mathbf{A}^{*}\right) \geq \operatorname{rank}\left(\mathbf{D}^{*}\right)-\operatorname{rank}\left(\mathbf{H}_{1}\right)=N-$ 1 [40]. However, if $\operatorname{rank}\left(\mathbf{A}^{*}\right)=N$, according to $\mathbf{A}^{*} \mathbf{W}_{1}^{*}=$ 0 , we will have $\mathbf{W}_{1}^{*}=0$, which is not an optimal solution to (12). Thus, in this case, $\operatorname{rank}\left(\mathbf{A}^{*}\right)=N-1$. So $\operatorname{rank}\left(\mathbf{W}_{1}^{*}\right)=$ 1 , and $\mathbf{W}_{1}^{*}=b \boldsymbol{\tau} \boldsymbol{\tau}^{H}$, where $\boldsymbol{\tau}$ spans the null space of $\mathbf{A}^{*}$.

If $l<N$, there must exist $\Pi \neq 0$ and $\mathbf{D}^{*} \Pi=0$. Then, we have

$$
\begin{aligned}
\boldsymbol{\pi}_{n}^{H} \mathbf{A}^{*} \boldsymbol{\pi}_{n} & =\boldsymbol{\pi}_{n}^{H}\left(\mathbf{H}_{1}-\mathbf{D}^{*}\right) \boldsymbol{\pi}_{n} \\
& =\boldsymbol{\pi}_{n}^{H} \mathbf{H}_{1} \boldsymbol{\pi}_{n}-\boldsymbol{\pi}_{n}^{H} \mathbf{D}^{*} \boldsymbol{\pi}_{n} \\
& =\left|\mathbf{h}_{1}^{H} \boldsymbol{\pi}_{n}\right|^{2} \geq 0,1 \leq n \leq N-l .
\end{aligned}
$$

Since $\mathbf{A}^{*} \leq 0$, it follows that $\left|\mathbf{h}_{1}^{H} \boldsymbol{\pi}_{n}\right|^{2}=0$, and it means that $\mathbf{H}_{1} \Pi=0$. So $\mathbf{A}^{*} \Pi=\left(\mathbf{H}_{1}-\mathbf{D}^{*}\right) \Pi=0$, and $\operatorname{rank}\left(\mathbf{A}^{*}\right) \geq \operatorname{rank}(\mathbf{D})-\operatorname{rank}\left(\mathbf{H}_{1}\right)=l-1$. Let $\Omega$ denote the orthogonal basis of the null space of $\mathbf{A}^{*}$, and $\operatorname{rank}(\Omega)=$ $N-\operatorname{rank}\left(\mathbf{A}^{*}\right) \leq N-l+1$. Since $\mathbf{A}^{*} \Pi=0$, $\Pi$ spans $(N-l)$ orthogonal dimensions of the null space of $\mathbf{A}^{*}$, i.e., $\operatorname{rank}(\Omega) \geq \operatorname{rank}(\Pi)=N-l$. If $\operatorname{rank}(\Omega)=N-l$, we have $\Omega=\Pi$ and $\mathbf{W}_{1}^{*}=\sum_{n=1}^{N-l} a_{n} \boldsymbol{\pi}_{n} \boldsymbol{\pi}_{n}^{H}$, where $a_{n} \geq 0$. In such case, $\mathbf{H}_{1} \mathbf{W}_{1}^{*}=0$, no unicasting information is transferred to user 1 since $\pi_{n}$ all lie in the null space of $\mathbf{H}_{1}$, i.e., $\mathbf{H}_{1} \boldsymbol{\Pi}=0$. Thus, there exists only one single subspace spanned by $\boldsymbol{\tau} \in \mathbb{C}^{N \times 1}$ of unit norm, which lies in the null space of $\mathbf{A}^{*}$, i.e., $\mathbf{A}^{*} \boldsymbol{\tau}=0$, and is orthogonal to the span of $\boldsymbol{\Pi}$, i.e., $\boldsymbol{\Pi}^{H} \boldsymbol{\tau}=0$. So we have $\boldsymbol{\Omega}=[\boldsymbol{\Pi}, \boldsymbol{\tau}]$ and $\operatorname{rank}(\boldsymbol{\Omega})=N-l+1$. Furthermore, any optimal solution to (12) can be expressed as (19).

If $\operatorname{rank}\left(\mathbf{W}_{1}^{*}\right)>1,\left(\hat{\mathbf{W}}_{1}^{*}, \hat{\mathbf{W}}_{0}^{*}\right)$, which is given in the second part of Proposition 2, is substituted into the objective function and constraints of (12), respectively, we have:

$$
\operatorname{tr}\left(\mathbf{H}_{1} \hat{\mathbf{W}}_{1}^{*}\right)=\operatorname{tr}\left[\mathbf{H}_{1}\left(\mathbf{W}_{1}^{*}-\sum_{n=1}^{N-l} a_{n} \boldsymbol{\pi}_{n} \boldsymbol{\pi}_{n}^{H}\right)\right]=\operatorname{tr}\left(\mathbf{H}_{1} \mathbf{W}_{1}^{*}\right)
$$

$$
\begin{aligned}
& \operatorname{tr}\left(\mathbf{H}_{k} \hat{\mathbf{W}}_{0}^{*}\right)-\gamma_{M} \operatorname{tr}\left(\mathbf{H}_{k} \hat{\mathbf{W}}_{1}^{*}\right) \\
& =\operatorname{tr}\left(\mathbf{H}_{k} \mathbf{W}_{0}^{*}\right)-\gamma_{M} \operatorname{tr}\left(\mathbf{H}_{k} \mathbf{W}_{1}^{*}\right)+\left(1+\gamma_{M}\right) \sum_{n=1}^{N-l} a_{n}\left|\boldsymbol{h}_{k}^{H} \boldsymbol{\pi}_{n}\right|^{2} \geq \gamma_{M} \sigma^{2}
\end{aligned}
$$




$$
\operatorname{tr}\left(\hat{\mathbf{W}}_{0}^{*}\right)+\operatorname{tr}\left(\hat{\mathbf{W}}_{1}^{*}\right)=\operatorname{tr}\left(\mathbf{W}_{0}^{*}\right)+\operatorname{tr}\left(\mathbf{W}_{1}^{*}\right) \leq P
$$

(23)-(25) indicate that the new solution $\left(\hat{\mathbf{W}}_{1}^{*}, \hat{\mathbf{W}}_{0}^{*}\right)$ can achieve the same optimal value of (12), while it always satisfies all the constraints of (12). So $\left(\hat{\mathbf{W}}_{1}^{*}, \hat{\mathbf{W}}_{0}^{*}\right)$ is also an optimal solution to (12) with $\operatorname{rank}\left(\hat{\mathbf{W}}_{1}^{*}\right)=1$. Proposition 2 is proved.

Thus, we can derive the optimal solution to problem (9) with rank-one matrix for $\mathbf{W}_{1}^{*}$ as follows. First, we solve problem (12) via CVX [41] and obtain the solution $\left(\mathbf{W}_{1}^{*}, \mathbf{W}_{0}^{*}\right)$. If $\operatorname{rank}\left(\mathbf{W}_{1}^{*}\right)=1$, the optimal unicasting and multicasting information beamforming vectors $\mathbf{w}_{1}^{*}$ and $\mathbf{w}_{0}^{*}$ for problem (9) can be obtained from the eigenvalue decomposition of $\mathbf{W}_{1}^{*}$ and $\mathbf{W}_{0}^{*}$, respectively. Otherwise, if $\operatorname{rank}\left(\mathbf{W}_{1}^{*}\right)>1$, a new solution $\left(\hat{\mathbf{W}}_{1}^{*}, \hat{\mathbf{W}}_{0}^{*}\right)$ with $\operatorname{rank}\left(\hat{\mathbf{W}}_{1}^{*}\right)=1$ can be constructed according to Proposition 2, and they are the optimal solution to problem (12). So, the rank-one relaxation on $\mathbf{W}_{1}^{*}$ in problem (12) results in no loss of optimality to problem (9), and given any $\gamma_{e}>0$, the value of $f\left(\gamma_{e}\right)$ can be obtained by solving problem (12).

Proposition 2 guarantees that problem (12) has a rankone optimal covariance solution $\mathbf{W}_{1}^{*}$, and if $\operatorname{rank}\left(\mathbf{W}_{1}^{*}\right)>$ 1 , the optimal value of problem (12) only serves as an upper bound on that of problem (9). So according to above analysis of Proposition 2, we have the following proposition.

Proposition 3 The optimal solution to problem (12) always satisfies that $\left(\mathbf{W}_{1}^{*}\right)=1$.

Proof According to (18), we have $\mathbf{D}^{*} \leq 0$ since $\mathbf{A}^{*} \leq 0$ and $\mathbf{H}_{1} \geq 0$. If $\operatorname{rank}\left(\mathbf{D}^{*}\right)=N$, it follows that $\operatorname{rank}\left(\mathbf{W}_{1}^{*}\right)=$ 1 is always satisfied according to Proposition 2. As a result, to show $\operatorname{rank}\left(\mathbf{D}^{*}\right)=N$, it is sufficient to verify that the maximum eigenvalue of $\mathbf{D}^{*}$ is negative, i.e., $\mathbf{D}^{*}<0$. Thus, in the following, we show by contradiction that the maximum eigenvalue of $\mathbf{D}^{*}$ must be negative.

Suppose that the maximum eigenvalue of $\mathbf{D}^{*}$ is zero. There is at least an $\mathbf{x} \in \mathbb{C}^{N \times 1} \neq 0$ such that:

$$
\mathbf{x}^{H} \mathbf{D}^{*} \mathbf{x}=0
$$

Since $\mathbf{A}^{*} \leq 0$ according to (18), we have

$$
\begin{aligned}
& \mathbf{x}^{H} \mathbf{A}^{*} \mathbf{x}=0 \\
& \mathbf{x}^{H} \mathbf{H}_{1} \mathbf{x}=0
\end{aligned}
$$

According to (15) and (18), we can derive the equation on the relationship between $\mathbf{B}^{*}$ and $\mathbf{D}^{*}$.

$$
\mathbf{D}^{*}=\sum_{k=2}^{K}\left[\alpha_{k}^{*}+\left(\gamma_{M}+1\right) \beta_{k}^{*}\right] \mathbf{H}_{k}+\left(\gamma_{M}+1\right) \beta_{1}^{*} \mathbf{H}_{1}-\mathbf{B}^{*}
$$

Substituting (28), (29), and (15) into (26), we can have

$$
\mathbf{x}^{H}\left\{\sum_{k=2}^{K}\left[\alpha_{k}^{*}+\left(\gamma_{M}+1\right) \beta_{k}^{*}\right] \mathbf{H}_{k}+\lambda \mathbf{I}\right\} \mathbf{x}=0
$$

Since $\lambda^{*}>0$ and $\alpha_{k}^{*} \geq 0, \beta_{k}^{*} \geq 0$ according to Proposition 1 and (17c), there is no non-zero solution to Eq. (30). Then, all the eigenvalues of $\mathbf{D}^{*}$ must be negative. Therefore $\operatorname{rank}\left(\mathbf{D}^{*}\right)=N$, and $\operatorname{rank}\left(\mathbf{W}_{1}^{*}\right)=1$. The Proposition 3 is proved.

To summarize, the algorithm for optimal solution to solve problem (9) is given in Algorithm 1.

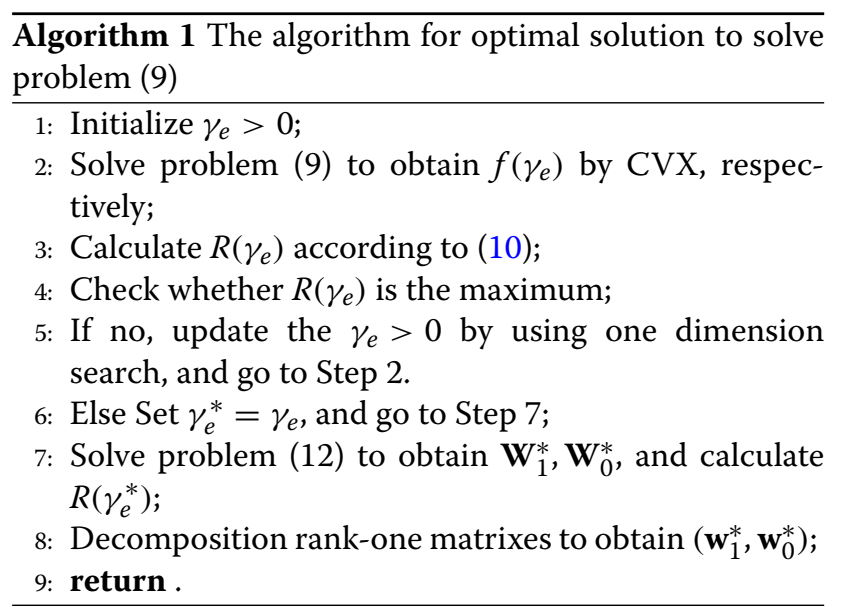

\section{The suboptimal solutions}

In order to reduce the computational complexity, we propose two suboptimal solutions for problem (9) based on zeroforcing (ZF) beamforming and maximal ratio transmission (MRT).

\subsection{The ZF-based suboptimal solution}

Assume that $K \leq N$, the ZF-based beamforming scheme can be used to reduce the interception by restricting unicasting information beamforming vector $\mathbf{w}_{1}$ to satisfy $\mathbf{h}_{k}^{H} \mathbf{w}_{1}=0, k=2, \cdots, K$, which simplifies the beamforming design.

Let $\mathbf{H}^{H}=\left[\boldsymbol{h}_{2}, \cdots, \mathbf{h}_{K}\right]^{H} \in \mathbb{C}^{(K-1) \times N}$, and the singular value decomposition of $\mathbf{H}$ is denoted as $\mathbf{H}^{H}=\mathbf{u} \Lambda \mathbf{v}^{H}=$ $\mathbf{u} \Lambda\left[\mathbf{v}_{1}, \mathbf{v}_{0}\right]^{H}$, where $\mathbf{u} \in \mathbb{C}^{(K-1) \times(K-1)}, \mathbf{v} \in \mathbb{C}^{N \times N}$ are the orthogonal left and right singular vectors of $\mathbf{H}$, respectively, which are both unitary matrices, $\Lambda \in \mathbb{C}^{(K-1) \times N}$ consists of $(K-1)$ positive singular values of $\mathbf{H}$. $\mathbf{v}_{0} \in$ $\mathbb{C}^{N \times(N-K+1)}$ is the last $(N-K+1)$ columns of $\mathbf{v}$ and forms an orthogonal basis for the null space of $\mathbf{H}$. The ZF-based precoding vector $\mathbf{w}_{1}$ can be expressed as $\mathbf{w}_{1}=\mathbf{v}_{0} \tilde{\mathbf{w}}_{1}$, where $\tilde{\mathbf{w}}_{1} \in \mathbb{C}^{(N-K+1) \times 1}$ denotes the new vector to be designed, and $\tilde{\mathbf{w}}_{0} \in \mathbb{C}^{N \times 1}$ is the ZF-based multicasting 
beamforming vector to be designed accordingly. In order to guarantee the feasibility of the ZF-based solution, we must have $K \leq N$.

Define $\tilde{\mathbf{H}}_{0}=\mathbf{v}_{0}^{H} \mathbf{h}_{1} \mathbf{h}_{1}^{H} \mathbf{v}_{0}$ and new variables $\tilde{\mathbf{W}}_{1}=\tilde{\mathbf{w}}_{1} \tilde{\mathbf{w}}_{1}^{H}$, $\tilde{\mathbf{w}}_{0}=\tilde{\mathbf{w}}_{0} \tilde{\mathbf{w}}_{0}^{H}$. Similar to the optimal solution, the SDR of problem (12) based on ZF is consequently formulated as:

$$
\begin{aligned}
& \max _{\tilde{\mathbf{W}}_{0} \geq 0, \tilde{\mathbf{W}}_{1} \geq 0} \operatorname{tr}\left(\tilde{\mathbf{H}}_{0} \tilde{\mathbf{W}}_{1}\right) \\
& \text { s.t. } \operatorname{tr}\left(\mathbf{H}_{k} \tilde{\mathbf{W}}_{0}\right) \geq \gamma_{M} \sigma^{2}, k=1, \cdots, K, \\
& \operatorname{tr}\left(\tilde{\mathbf{W}}_{0}\right)+\operatorname{tr}\left(\tilde{\mathbf{W}}_{1}\right) \leq P,
\end{aligned}
$$

Problem (31) can be solved by using the technique of CVX, and then the corresponding solution also can be derived according to Proposition 2. Different from the optimal scheme, the ZF-based scheme significantly reduces the computational complexity without the onedimension search. The algorithm for suboptimal solution based on ZF is summarized as Algorithm 2.

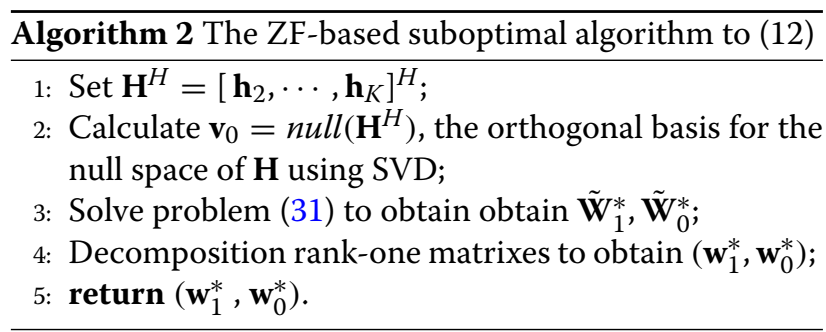

\subsection{The MRT-based suboptimal solution}

In the second suboptimal solution, with the application of MRT principle and given $\gamma_{e}$, the beamforming vectors are artificially designed to improve the effective channel gain of unicasting information [30], i.e., $\mathbf{w}=\mathbf{h}_{1} /\left\|\mathbf{h}_{1}\right\|$, and the suboptimal solution works for arbitrary value of $N$. It means that $\mathbf{w}_{1}=\sqrt{\alpha_{U}} \mathbf{w}, \mathbf{w}_{0}=\sqrt{\alpha_{M}} \mathbf{w}$ in (9), where $\alpha_{M}, \alpha_{U}$ are the power of multicasting and unicasting information which are designed to satisfy transmission power constraint of the BS, i.e., $\alpha_{M}+\alpha_{U} \leq P$. So the MRT-based secrecy unicasting rate maximization problem is transformed into a power allocation problem, which can be expressed as:

$$
\begin{aligned}
& \max _{\alpha_{U}>0, \alpha_{M}>0} \alpha_{U}\left|\mathbf{h}_{1}^{H} \mathbf{w}\right|^{2} \\
& \text { s.t. } \alpha_{U}\left|\mathbf{h}_{k}^{H} \mathbf{w}\right|^{2} \leq \gamma_{e} \sigma^{2}, k=2, \cdots, K \\
& \alpha_{M}\left|\mathbf{h}_{k}^{H} \mathbf{w}\right|^{2} \geq \gamma_{M}\left(\alpha_{U}\left|\mathbf{h}_{k}^{H} \mathbf{w}\right|^{2}+\sigma^{2}\right), k=1, \cdots, K
\end{aligned}
$$

$$
\alpha_{M}+\alpha_{U} \leq P,
$$

As mentioned before, the constraint (32d) must be satisfied with equality, i.e., $\alpha_{M}+\alpha_{U}=P$. So with given $\gamma_{e}>0$, from (32b) and (32c), we can derive the feasible set of power allocation for unicasting information as:

$$
\alpha_{U} \in\left\{0, \min _{2 \leq k \leq K}\left[\frac{P\left|\mathbf{h}_{k}^{H} \mathbf{w}\right|^{2}-\gamma_{M} \sigma^{2}}{\left|\mathbf{h}_{k}^{H} \mathbf{w}\right|^{2}\left(\gamma_{M}+1\right)}, \frac{\gamma_{e} \sigma^{2}}{\left|\mathbf{h}_{k}^{H} \mathbf{w}\right|^{2}}\right]\right\}
$$

Since the objective function of (32a) is a monotonically increasing function about $\alpha_{U}$, the optimal solution can be expressed as:

$$
\alpha_{U}^{*}=\min _{2 \leq k \leq K}\left[\frac{P\left|\mathbf{h}_{k}^{H} \mathbf{w}\right|^{2}-\gamma_{M} \sigma^{2}}{\left|\mathbf{h}_{k}^{H} \mathbf{w}\right|^{2}\left(\gamma_{M}+1\right)}, \frac{\gamma_{e} \sigma^{2}}{\left|\mathbf{h}_{k}^{H} \mathbf{w}\right|^{2}}\right]
$$

Thus, the secrecy rate of unicasting based on MRT also can be obtained by one-dimension search over $\gamma_{e}>0$, just as the optimal solution. The detailed steps of the MRTbased scheme are presented as Algorithm 3.

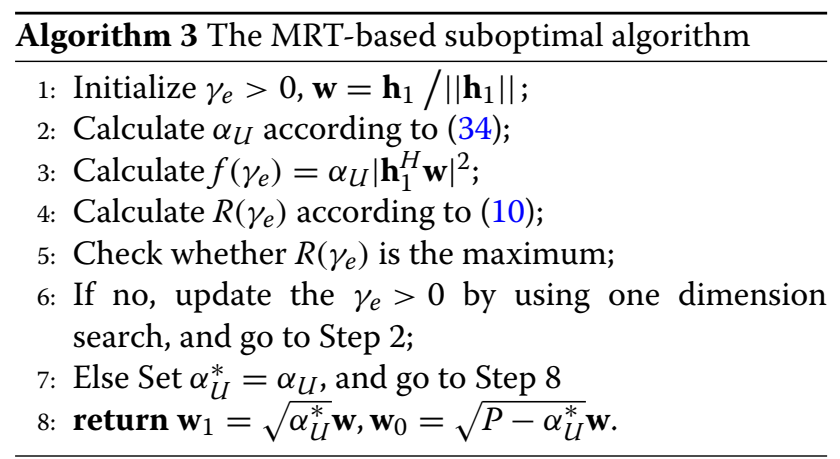

\section{Simulation results and discussions}

In this section, we numerically evaluate the performance of the proposed optimal and suboptimal schemes in the NOMA-enabled multicast-unicast transmission system, in which the BS is equipped with $N$ antennas, and servers $K$ users. Combining the feasibility of the ZF-based scheme with the facility cost in practice, we set $N=K$ and $\sigma^{2}=-50 \mathrm{dBm}, P=20 \mathrm{dBm}$ in the simulations. We assume that signal attenuation from BS to the special user is $55 \mathrm{~dB}$, corresponding to an identical distance of $15 \mathrm{~m}$, and the signal attenuations from BS to other users are no less than $65 \mathrm{~dB}$, corresponding to an identical distance of $20 \mathrm{~m}$ considering the user scheduling. The channel vectors are randomly generated from i.i.d. Rayleigh fading. All simulation results are achieved over 1000 random channel realization.

First, we check that given $\gamma_{e}>0$, whether problem (11) can be optimally solved. Figure 2 shows the plot about the function $R\left(\gamma_{e}\right)=\log _{2}\left[\frac{\sigma^{2}+f\left(\gamma_{e}\right)}{\sigma^{2}\left(1+\gamma_{e}\right)}\right]$ over $\gamma_{e}>0$ with $N=K=4$. We adopt one-dimension search to find 


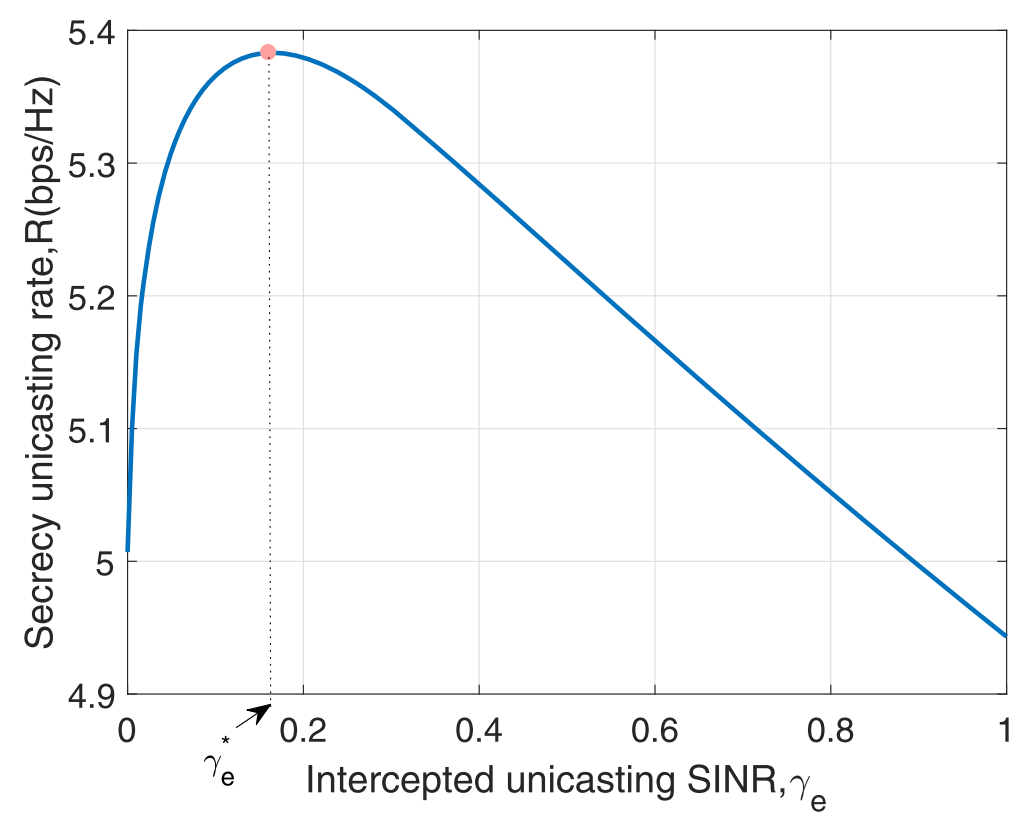

Fig. 2 Uniqueness of $\gamma_{e}^{*}$ in the multicast-unicast transmission system

the maximum of $R\left(\gamma_{e}\right)$, and the uniqueness of $\gamma_{e}^{*}$ guarantees the validity of one-dimension search. Meanwhile, given $\gamma_{e}>0$, the corresponding solution $\left(\boldsymbol{w}_{0}, \boldsymbol{w}_{1}\right)$ of problem (9) can be derived. Under such particular setup, there is only one single maximum point in the plotted function. Actually, we cannot verify analytically the concavity or even the quasi-concavity of the function. From Fig. 2, we can observe that under such a situation, there is only one single maximal value $\gamma_{e}^{*}$, and many others used in simulations of algorithm 1 and 3 also have their unique points.

Next, with the transmission power of the BS $P=20$ $\mathrm{dBm}$ and the target rate of multicasting information $R_{M}=$ $1 \mathrm{bps} / \mathrm{Hz}$, Fig. 3 compares the secrecy rate of unicasting

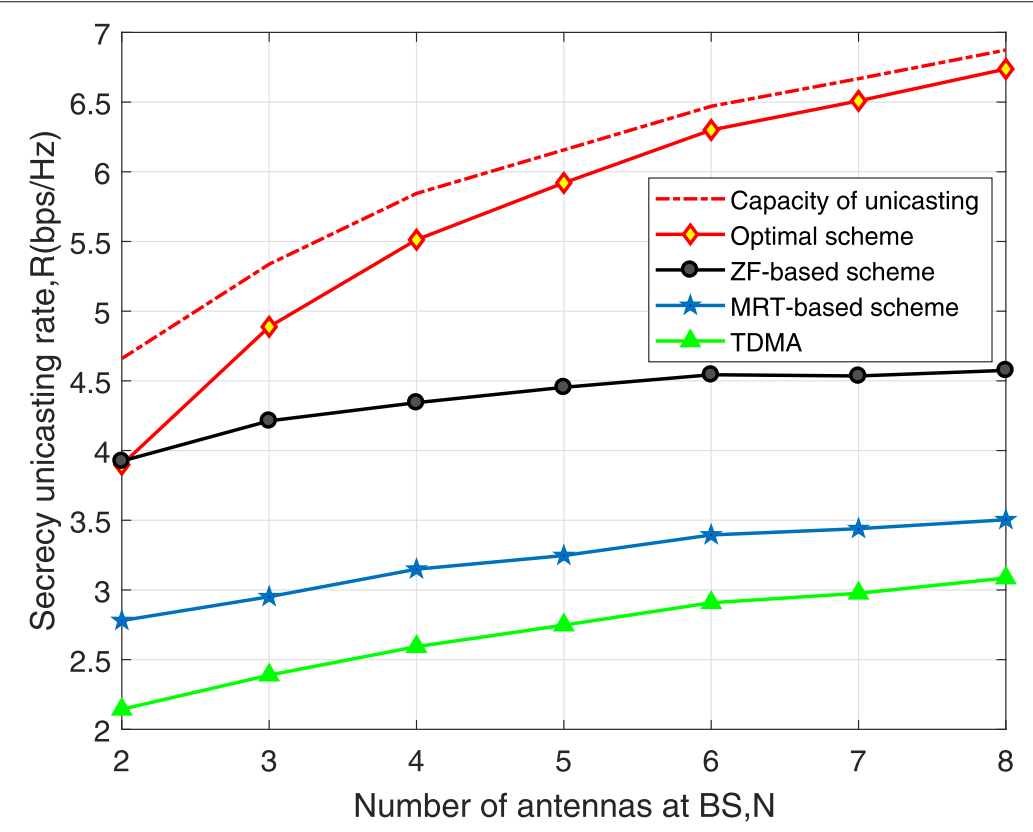

Fig. 3 Secrecy unicasting rate versus the number of users with $P=20 \mathrm{dBm}$ 


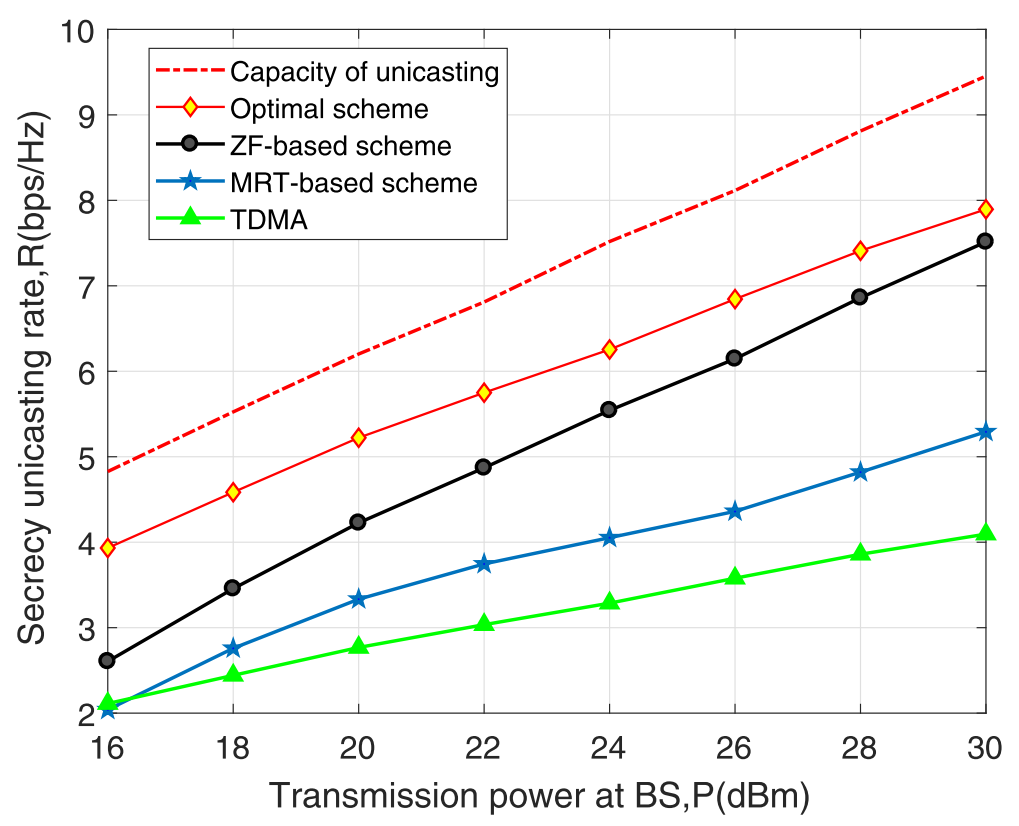

Fig. 4 Secrecy rate of unicasting versus the transmission power with $R_{M}=1 \mathrm{bps} / \mathrm{Hz}$

information versus the number of antennas at the BS, which is set to be equal to the number of users in all simulations. The capacity of unicasting rate is also showed simultaneously under ideal conditions without the interception of unicasting.

Without loss of generality, time division multiple access (TDMA) is used as a representative of OMA, in which the multicasting and unicasting information are separately transmitted in different time slot. From the simulation results showed in the Fig. 3, the security performance of our proposed NOMA assisted system is undoubtedly better than that of TDMA system, and the performance of our proposed schemes is better with the growth of the number of antennas. Although the number of users also increases, the unicasting rate can be improved by proper beamforming design with more antennas at BS, and especially the optimal scheme has a significant improvement on the security performance. And the performance of optimal scheme is much closer to the capacity of the unicasting.

Then, we illustrate the secrecy rate of unicasting for our proposed optimal and suboptimal schemes, including TDMA scheme, against the transmission power at the BS in Fig. 4. The capacity of unicasting rate is also compared versus the transmission power of the BS under ideal conditions without the interception of unicasting. It is observed that the optimal and suboptimal strategies can achieve higher secrecy rate than TDMA, and with more power of transmit supply, the performance of the ZFbased scheme in algorithm 2 is close to that of the optimal scheme.
In order to compare the computational complex of proposed schemes, we list the running time of proposed optimal and suboptimal solution in Table 1 with the average time over 100 random channel realization. Regardless of the computer hardware, we can find that the running time of optimal scheme is tremendously large because of the matrix optimization and the one-dimension search, especially with more users.

At last, we show in Fig. 5 the impact of the target multicasting rate, $R_{M}$, on the secrecy rate of unicasting achieved for all proposed solutions, with fixed transmission power at the $\mathrm{BS} P=25 \mathrm{dBm}$ and $P=30 \mathrm{dBm}$, respectively. It is observed that the curves of all solutions decreases with the target rate of multicasting. The reason is that more power is allocated to transmit multicasting information so as to meet the requirement of QoS. However, increasing the transmission power can improve the secrecy unicasting rate.

\section{Conclusion}

This paper has investigated the security problem in a UAV-aided multiuser network with multicast-unicast transmission assisted by NOMA. Using the two-stage

Table 1 The running time of three schemes (unit, second)

\begin{tabular}{lllllll}
\hline$K$ & 3 & 4 & 5 & 6 & 7 & 8 \\
Optimal & 7.6973 & 8.0412 & 8.4395 & 8.7480 & 9.0384 & 9.6459 \\
ZF & 0.3100 & 0.3195 & 0.3280 & 0.3367 & 0.3386 & 0.3810 \\
MRT & 0.0310 & 0.0076 & 0.0101 & 0.0120 & 0.0871 & 0.0901 \\
\hline
\end{tabular}




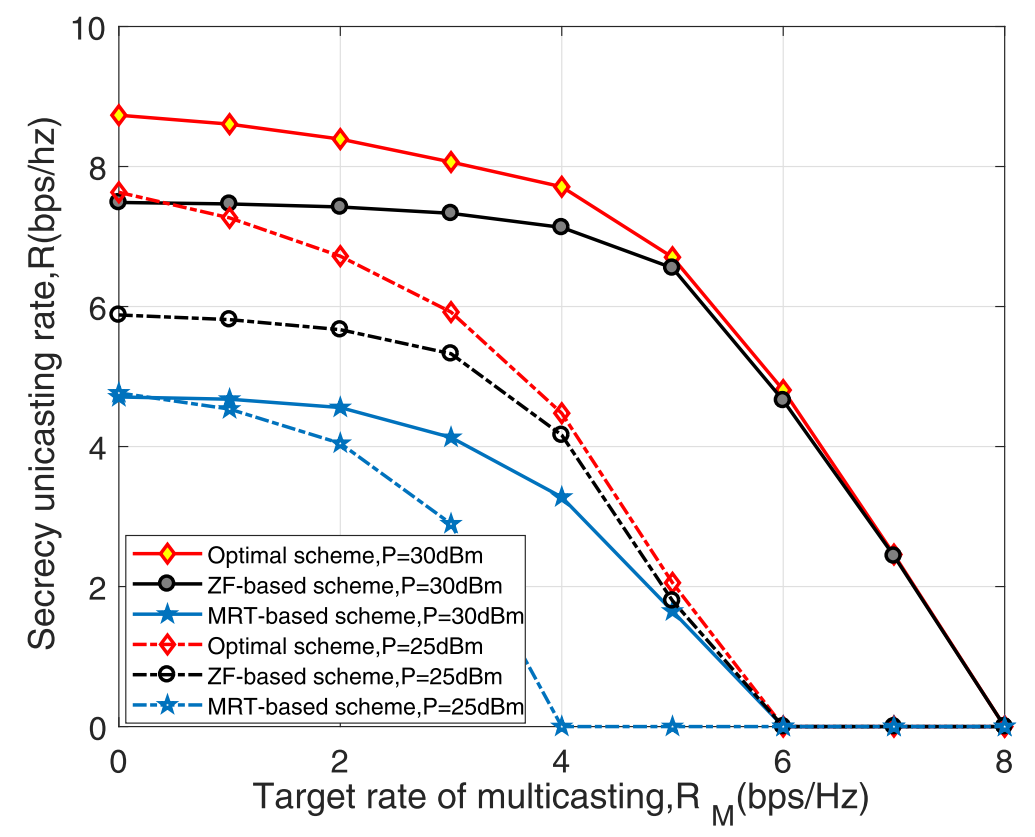

Fig. 5 Secrecy unicasting rate versus the target rate of multicasting with $K=5$

procedure, we can optimally solve the non-convex design problem by applying the technique of SDR. We also show that SDR here has no loss of optimality. Two suboptimal solutions with lower complexity than the optimal solution are also presented based on ZF and MRT. Experimental results show that the new approach of placement problem improves the security performance of UAV communication systems.

\section{Acknowledgement}

Not applicable.

\section{Abbreviations}

UAV: Unmanned aerial vehicle; NOMA: Non-orthogonal multiple access; SIC: Successive interference cancellation; BS: Base station; LoS: Line-of-sight; SDR Semidefinite relaxation; MRT: Maximal ratio transmission

\section{Authors' contributions}

Chuan Yin is the main author of the current paper. Chuan Yin contributed to the development of the ideas, design of the study, theory, result analysis, and article writing. Leibing Yan carried out the experimental work and the data collection and interpretation and finished the analysis and interpretation of data and drafted the manuscript. All authors read and approved the final manuscript.

\section{Funding}

This work is supported by the National Natural Science Foundation of China under grant numbers 61801153, State Key Laboratory of Millimeter Waves under grant numbers K202012, Natural Science Foundation of Zhejiang Province (LQY2OF010001) and Doctoral Research Fund of Henan Institute of Technology (KQ1852).

\section{Availability of data and materials}

Data sharing is not applicable to this article as no datasets were generated or analyzed during the current study.

\section{Competing interests}

The authors declare that they have no competing interests.

\section{Author details}

${ }^{1}$ School of electronic and information, Hangzhou Dianzi University, 310018, Hangzhou, China. ${ }^{2}$ School of Electronic Information Engineering, Henan Institute of Technology, 453003, Xinxiang, China.

Received: 21 December 2019 Accepted: 6 March 2020

Published online: 20 April 2020

\section{References}

1. Y. Zeng, R. Zhang, T.J. Lim, Wireless communications with unmanned aerial vehicles: Opportunities and challenges. IEEE Commun. Mag. 54(5), 36-42 (2016)

2. C. Li, H. J. Yang, F. Sun, J. M. Cioffi, L. Yang, Adaptive overhearing in two-way multi-antenna relay channels. IEEE Sig. Process. Lett. 23(1), 117-120 (2016)

3. F. Zhou, Y. Wu, R. Q. Hu, Y. Qian, Computation rate maximization in UAV-enabled wireless powered mobile-edge computing systems. IEEE J. Sel. Areas Commun. 36(9), 1927-1941 (2018)

4. C. Li, H. J. Yang, F. Sun, J. M. Cioffi, L. Yang, Multiuser overhearing for cooperative two-way multiantenna relays. IEEE Trans. Veh. Technol. 65(5), 3796-3802 (2016)

5. Z. Ding, Z. Yang, P. Fan, H. V. Poor, On the performance of non-orthogonal multiple access in $5 \mathrm{G}$ systems with randomly deployed users. IEEE Sig. Process. Lett. 21(12), 1501-1505 (2014)

6. W. Wu, F. Zhou, R. Q. Hu, B. Wang, Energy-efficient resource allocation for secure NOMA-enabled mobile edge computing networks. IEEE Trans. Commun. 68(1), 493-505 (2019). https://doi.org/10.1109/tcomm.2019. 2949994

7. F. Zhou, Y. Wu, R. Q. Hu, Y. Wang, K. Wong, Energy-efficient NOMA heterogeneous cloud radio access networks. IEEE Netw. 32(2), 152-160 (2018)

8. Y. Saito, Y. Kishiyama, A. Benjebbour, T. Nakamura, A. Li, K. Higuchi, in Proc. IEEE Veh. Tech. Conf. (VTC Spring). Non-orthogonal multiple access (NOMA) for cellular future radio access (IEEE, 2013), pp. 1-5. https://doi.org/10. 1109/vtcspring.2013.6692652

9. C. Li, F. Sun, J. M. Cioffi, L. Yang, Energy efficient MIMO relay transmissions via joint power allocations. IEEE Trans. Circ. Syst. 61(7), 531-535 (2014)

10. P. Deng, B. Wang, W. Wu, T. Guo, Transmitter design in MISO-NOMA system with wireless-power supply. IEEE Commun. Lett. 22(4), 844-847 (2018) 
11. C. Li, S. Zhang, P. Liu, F. Sun, J. M. Cioffi, L. Yang, Overhearing protocol design exploiting inter-cell interference in cooperative green networks. IEEE Trans. Veh. Technol. 65(1), 441-446 (2016)

12. W. Wu, F. Zhou, R. Q. Hu, B. Wang, Energy-Efficient Resource Allocation for Secure NOMA-Enabled Mobile Edge Computing Networks. IEEE Trans. Commun. 68(1), 493-505 (2020). https://doi.org/10.1109/TCOMM.2019. 2949994

13. J. Cui, Y. Liu, Z. Ding, P. Fan, A. Nallanathan, Optimal user scheduling and power allocation for millimeter wave NOMA systems. IEEE Trans. Wireless Commun. 17(3), 1502-1517 (2018)

14. W. Wu, F. Zhou, P. Li, P. Deng, B. Wang, V. C. M. Leung, in ICC 2019-2019 IEEE International Conference on Communications (ICC). Energy efficient secure NOMA-enabled mobile edge computing networks (IEEE, 2019). https://doi.org/10.1109/icc.2019.8761823

15. F. Zhou, Y. Wu, Y. Liang, Z. Li, Y. Wang, K. Wong, State of the art, taxonomy, and open issues on cognitive radio networks with NOMA. IEEE Wirel. Commun. 25(2), 100-108 (2018)

16. C. Li, P. Liu, C. Zou, F. Sun, J. M. Cioffi, L. Yang, Spectral-efficient cellular communications with coexistent one- and two-hop transmissions. IEEE Trans. Veh. Technol. 65(8), 6765-6772 (2016)

17. W. Wu, Y. Wang, J. Mo, J. Liu, in EURASIP Journal on Wireless Communications and Networking. Robust Proactive Eavesdropping in UAV-Enabled Wireless Communication Networking (Springer, 2019). https://doi.org/10.1186/s13638-019-1599-6

18. F. Zhou, Y. Wu, Y. Liang, Z. Li, Y. Wang, K. Wong, State of the art, taxonomy, and open issues on NOMA in cognitive radio networks. IEEE Wirel. Commun. 25(2), 100-108 (2018)

19. Sharma P.K., D. I. Kim, in Proc. IEEE Globecom Workshops (GC Wkshps). UAV-enabled downlink wireless system with non-orthogonal multiple access (IEEE, 2017). https://doi.org/10.1109/glocomw.2017.8269066

20. Q. Wu, J. Xu, R. Zhang, Capacity characterization of UAV-enabled two-user broadcast channel. IEEE J. Sel. Areas Commun. 36(9), 1955-1971 (2018)

21. M. F. Sohail, C. Y. Leow, S. Won, Non-orthogonal multiple access for unmanned aerial vehicle assisted communication. IEEE Access. 6, 22716-22727 (2018)

22. M. M. Azari, F. Rosas, K.-C. Chen, S. Pollin, Ultra reliable UAV communication using altitude and cooperation diversity. IEEE Trans. Commun. 66(1), 330-344 (2018)

23. Z. Ding, P. Fan, H. V. Poor, Impact of user pairing on $5 \mathrm{G}$ nonorthogonal multiple-access downlink transmissions. IEEE Trans. Veh. Technol. 65(8), 6010-6023 (2016)

24. U. Sethakaset, S. Sun, in 21 st Annual IEEE International Symposium on Personal, Indoor and Mobile Radio Communications. Sum-rate maximization in the simultaneous unicast and multicast services with two users (IEEE, 2010). https://doi.org/10.1109/pimrc.2010.5671672

25. W. Wu, B. Wang, Z. Deng, H. Zhang, Secure beamforming for full-duplex wireless powered communication systems with self-energy recycling. IEEE Wirel. Commun. Lett. 6(2), 146-149 (2017)

26. H. Ly, T. Liu, Y. Liang, Multiple-input multiple-output Gaussian broadcast channels with common and confidential messages. IEEE Tran. Inf. Theory. 56(11), 5477-5487 (2010)

27. R. Liu, T. Liu, H. V. Poor, S. Shamai, in 2010 IEEE International Symposium on Information Theory. MIMO Gaussian broadcast channels with confidential and common messages (IEEE, 2010). https://doi.org/10.1109/isit.2010. 5513775

28. F. Zhou, Z. Chu, H. Sun, R. Q. Hu, L. Hanzo, Artificial noise aided secure cognitive beamforming for cooperative MISO-NOMA using SWIPT. IEEE J. Sel. Areas Commun. 36(4), 918-931 (2018)

29. B. Kim, S. Lim, H. Kim, S. Suh, J. Kwun, S. Choi, C. Lee, S. Lee, D. Hong, in MILCOM 2013 - 2013 IEEE Military Communications Conference. Non-orthogonal multiple access in a downlink multiuser beamforming system (IEEE, 2013). https://doi.org/10.1109/milcom.2013.218

30. Z. Ding, Z. Zhao, M. Peng, H. V. Poor, On the spectral efficiency and security enhancements of NOMA assisted multicast-unicast streaming. IEEE Trans. Commun. 65(7), 3151-3163 (2017)

31. W. Mei, Z. Chen, J. Fang, GSVD-based precoding in MIMO systems with integrated services. IEEE Sig. Process. Lett. 23(11), 1528-1532 (2016)

32. E. Karipidis, N. Sidiropoulos, Z.-Q. Luo, Quality of service and Max-Min fair transmit beamforming to multiple cochannel multicast groups. IEEE Trans. Sig. Process. 56(3), 1268-1279 (2008)
33. H.-H. Chen, L. Wang, Physical layer security for next generation wireless networks: Theories, technologies, and challenges. IEEE Comm. Surv. Tuts. 19(1), 347-376 (2017)

34. W.-C. Liao, T.-H. Chang, W.-K. Ma, C.-Y. Chi, QoS-based transmit beamforming in the presence of eavesdroppers: an optimized artificial-noise-aided approach. IEEE Trans. Sig. Process. 59(3), 1202-1216 (2011)

35. P. Gover, A. Sahai, in 2010 IEEE International Symposium on Information Theory. Shannon meets Tesla: wireless information and power transfer (IEEE, 2010). https://doi.org/10.1109/isit.2010.5513714

36. Z.-Q. Luo, W.-K. Ma, A. M.-C. So, Y. Ye, S. Zhang, Semidefinite relaxation of quadratic optimization problems. IEEE Sig. Process. Mag. 27(3), 20-34 (2010)

37. L. Zhang, R. Zhang, Y. C. Liang, Y. Xin, S. Cui, On the relationship between the multi-antenna secrecy communications and cognitive radio communications. IEEE Trans. Commun. 58(6), 1877-1886 (2010)

38. Y. Huang, D. P. Palomar, Rank-constrained separable semidefinite programming with applications to optimal beamforming. IEEE Trans. Sig. Process. 58(2), 664-678 (2010)

39. S. Boyd, L. Vandenberghe, Convex Optimization. (Cambridge Univ. Press, Cambridge U.K., 2004)

40. L. Liu, R. Zhang, K.-C. Chua, in 2013 IEEE Global Communications Conference (GLOBECOM). Secrecy wireless information and power transfer with MISO beamforming (IEEE, 2013). https://doi.org/10.1109/glocom.2013.6831340

41. M. Grant, S. Boyd, CVX: Matlab Software for Disciplined Convex Programming, Version 1.21 (2011). http://download.csdn.net/detail/ herosofearth/9631094

\section{Publisher's Note}

Springer Nature remains neutral with regard to jurisdictional claims in published maps and institutional affiliations.

\section{Submit your manuscript to a SpringerOpen ${ }^{\circ}$ journal and benefit from:}

- Convenient online submission

- Rigorous peer review

- Open access: articles freely available online

- High visibility within the field

- Retaining the copyright to your article

Submit your next manuscript at $>$ springeropen.com 